\title{
BMJ Open Evaluation of a stand-alone mobile mindfulness app in people experiencing infertility: the protocol for an exploratory randomised controlled trial (MoMiFer-RCT)
}

\author{
Tessy Boedt (D) , ${ }^{1,2}$ Nele Willaert, ${ }^{3}$ Sharon Lie Fong, ${ }^{4,5}$ Eline Dancet (D) ,,5 \\ Carl Spiessens, ${ }^{4,5}$ Filip Raes, ${ }^{2,3}$ Christophe Matthys (1) , ${ }^{1,6}$ Katleen Van der Gucht ${ }^{2,3}$
}

To cite: Boedt T, Willaert $\mathrm{N}$, Lie Fong $\mathrm{S}$, et al. Evaluation of a stand-alone mobile mindfulness app in people experiencing infertility: the protocol for an exploratory randomised controlled trial (MoMiFer-RCT). BMJ Open 2022;12:e050088. doi:10.1136/ bmjopen-2021-050088

- Prepublication history and additional supplemental material for this paper are available online. To view these files, please visit the journal online (http://dx.doi.org/10.1136/ bmjopen-2021-050088)

Received 09 February 2021 Accepted 24 December 2021

Check for updates

(C) Author(s) (or their employer(s)) 2022. Re-use permitted under CC BY-NC. No commercial re-use. See rights and permissions. Published by BMJ.

For numbered affiliations see end of article.

Correspondence to

Tessy Boedt;

tessy.boedt@kuleuven.be

\section{ABSTRACT}

Introduction Infertility and its treatment bring a considerable emotional burden. Increasing evidence demonstrates the effectiveness of smartphone-delivered mindfulness apps for reducing symptoms of emotional distress in both clinical and non-clinical populations. Evidence on this topic in women, men and couples experiencing infertility is currently under-represented. The aim of the MoMiFer study is, therefore, to investigate the efficacy of a stand-alone mobile mindfulness app on symptoms of emotional distress and fertility-related quality of life in people experiencing infertility.

Methods and analysis This study is an exploratory randomised controlled trial (RCT) with open enrollment. The primary outcomes are symptoms of emotional distress and fertility-related quality of life. Secondary outcomes are mindfulness skills, repetitive negative thinking, self-compassion, user-rated quality of the stand-alone mobile mindfulness app and use of the app. Experience sampling method and standardised self-report questionnaires are combined within a repeated measures design to measure the effects of the stand-alone mobile mindfulness app on the primary and secondary outcomes, apart from the use of the app. The latter will be evaluated through app tracking. People, including women, men and couples, experiencing infertility $(n=60)$ will be randomised to an intervention group receiving the standalone mobile mindfulness app for 3 months or a wait-list control group. The app follows the format and content of Mindfulness-Based Stress Reduction. Data will be collected at baseline, at 1.5 months and 3 months after randomisation. Analysis will be according to intention to treat and based on general linear modelling and multilevel mixed-effects modelling.

Ethics and dissemination This study received approval from the Medical Ethical Committee of the Leuven University Hospital (Belgium). The findings of this exploratory RCT will be disseminated through presentations at public lectures, scientific institutions and meetings, and through peer-reviewed scientific articles.

Trial registration number NCT04143828.
Strengths and limitations of this study

- Development of the stand-alone mobile mindfulness app is evidence based, which includes involvement of patients with infertility and healthcare professionals.

- In the moment measurements through experience sampling method are combined with self-report questionnaires.

- Participants include couples and individuals within a repeated measures design.

- This is an open-label study where only the statistician is blinded.

- Wait-list control group is used which may inflate the estimated effect.

\section{INTRODUCTION}

Infertility is defined as the inability to achieve a clinical pregnancy after 1 year of regular unprotected sexual intercourse. ${ }^{1}$ An estimated 48.5 million couples suffer from infertility worldwide. ${ }^{2}$ Consequently, assisted reproductive technology (ART) utilisation for infertility treatment including in vitro fertilisation (IVF) with or without intracytoplasmic sperm injection (ICSI) continues to increase. ${ }^{34}$ Approximately one-third of IVF with or without ICSI cycles result in a clinical pregnancy and subsequent life birth in circa $20 \%{ }^{3}$

Infertility and its treatment result in considerable emotional burden for women, men and couples. ${ }^{5-7}$ Proportionately more female than male partners report this burden, ${ }^{8-11}$ which can be seen across distinct cultures. ${ }^{11}$ Moreover, several studies have shown that this emotional burden may be an important reason for couples to terminate ART without achieving a live birth. ${ }^{12} 13$ Mindfulnessbased interventions (MBIs), such as 
Mindfulness-Based Stress Reduction (MBSR), ${ }^{14}$ have been found to reduce psychological symptoms, and improve well-being in both clinical and non-clinical populations. ${ }^{15-18}$ Multiple meta-analyses show that the strongest effects of MBIs were seen on symptoms of stress, depression and anxiety. ${ }^{19-22}$

Different supportive psychosocial interventions have been developed for people with infertility across the treatment cycle, ${ }^{23}$ including MBIs targeting women with infertility. $^{21} 24$ A systematic review covering face-to-face MBIs found small to moderate reductions in anxiety and depression for women with infertility, ${ }^{25}$ which appear to be maintained over a longer period of time. ${ }^{26}$ Improvements in mindfulness skills, ${ }^{2427}$ quality of life ${ }^{202128}$ and stress symptoms ${ }^{2027}$ have been identified as well. However, most findings solely focus on women with infertility and exclude the male partner. ${ }^{25}$

Research regarding the effectiveness of online and smartphone-delivered self-administered interventions as a tool to support mental health is growing. ${ }^{16-31}$ What is key here, is that such tools make low-intensive psychological help easily accessible for a large audience at lowcost. A recent meta-analysis found promising results for smartphone-delivered mindfulness meditation apps in clinical and non-clinical populations for multiple psychological outcomes including anxiety, depressive symptoms, quality of life and perceived stress. ${ }^{30}$ Moreover, smartphone-delivered apps including mindfulness and acceptance components seem to improve both components. ${ }^{32}$ Given the emotional burden of infertility $^{5}$ and its treatment, ${ }^{6733}$ and the time cost of fertility treatment, people experiencing infertility require easily accessible, low intensive strategies to improve their mental health. ${ }^{34}$

In this study, we want to target symptoms of emotional distress and quality of life by offering a stand-alone mobile mindfulness app to support people experiencing infertility.

\section{METHODS AND ANALYSIS}

This protocol was written according to Standard Protocol Items: Recommendations for Interventional Trials. ${ }^{35}$

\section{Aim}

The overall aim of the MoMiFer-study is to examine the effect of a stand-alone mobile mindfulness app on symptoms of emotional distress (symptoms of stress, anxiety and depression) and quality of life in people, including women, men and couples, experiencing infertility.

The objectives are as follows:

- To evaluate the impact of a stand-alone mobile mindfulness app (MoMiFer-app) on symptoms of emotional distress and quality of life in comparison with a control condition. We hypothesise a reduction in symptoms of emotional distress and an increase in quality of life in the experimental group as compared with the control group.

- To explore whether changes in symptoms of emotional distress are associated with improvements in mindfulness skills and self-compassion on the one hand, and a reduction in repetitive negative thinking (RNT) on the other hand.

\section{Study design and timing}

The MoMiFer-randomised controlled trial (RCT) is an exploratory study. ${ }^{36}$ This trial uses a parallel group design, with allocation ratio 1:1. The experimental group has immediate access to the MoMiFer-app for 3 months. The control group is wait-listed for 3 months after which access to the MoMiFer-app is received. The MoMiFerstudy is a repeated measures design and will result in 3 waves of time-series data for each participant. Recruitment through open enrolment started on 24 November 2019. Figure 1 presents an overview of the study design.

\section{Recruitment and study setting}

Participants are recruited through two Belgian non-profit organisations for people experiencing infertility 'De Verdwaalde Ooievaar' and 'Kinderwens'. Both organisations,

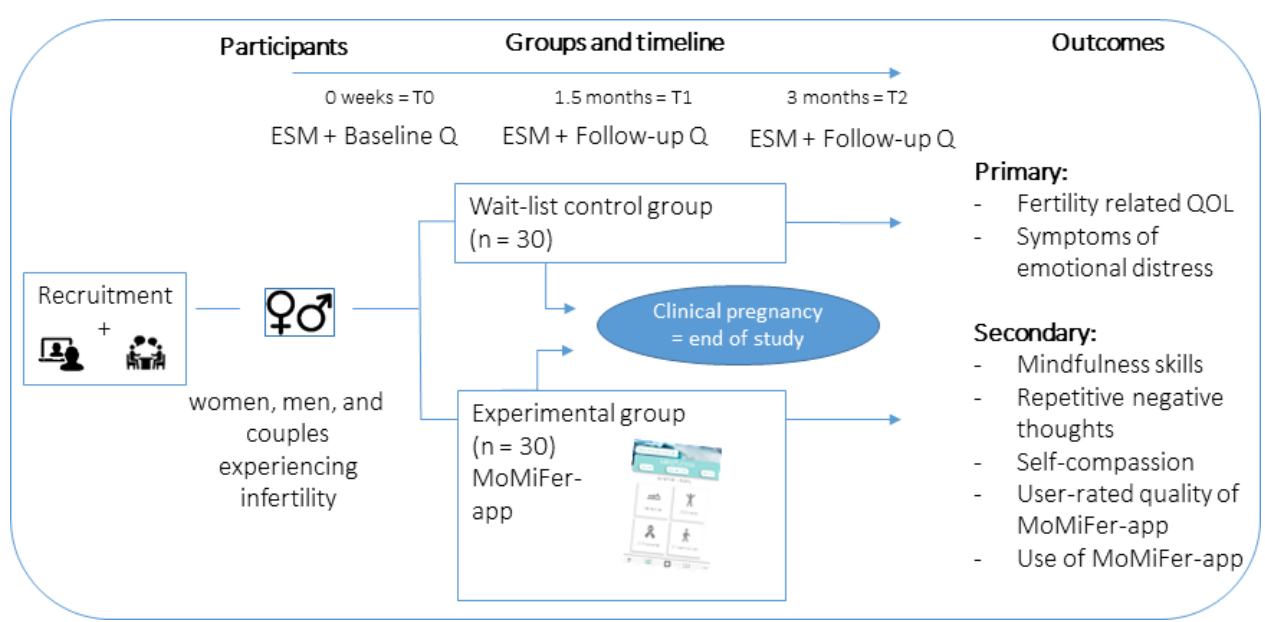

Figure 1 Overview of the MoMiFer-RCT. ESM, experience sampling method; QOL, quality of life; RCT, randomised controlled trial. 
together with 'PraxisP', promote this study by posting a flyer on their websites and social media accounts at regular times to achieve the targeted sample size. In addition, the study is promoted via social media. Participants who are interested receive detailed information from our researcher sending the informed consent (online supplemental material 1) through mail.

\section{Eligibility criteria}

Inclusion criteria are: women, men and couples speaking and understanding Dutch, and experiencing infertility, defined as the failure to achieve a clinical pregnancy after 12 months or more of regular unprotected sexual intercourse, ${ }^{1}$ aged $18-43$, and who are in the possession of a smartphone. Couples undergoing fertility treatment at time of recruitment will also be eligible. Concomitant care (eg, acupuncture, fertility counsellor) during the study is allowed.

\section{Randomisation, blinding and treatment allocation}

A computer-generated blocked randomisation procedure via a password-protected website (1:1 allocation ratio) with randomly varying the block size after baseline assessment is applied. This guarantees the allocation concealment to the recruiter. Due to the study design, neither the participants, nor the researchers are blinded for treatment allocation, expect for the statistician (data-analysis is done by third party).

\section{Interventions}

Through randomisation participants are assigned to either an experimental group which receives immediate access to the MoMiFer-app for 3 months, or to a wait-list condition. When assigned to the control group, participants gain access to the MoMiFer-app after 3 months. If a clinical pregnancy occurs during the trial, participation to the study ends. The MoMiFer-app includes mindfulness exercises following the format and content of MBSR. ${ }^{143738}$ They were originally developed as part of the theory-based and evidence-based 'Prelife programme', ${ }^{39}$ which is based on prior consultation and feedback from patients with infertility, and expert opinion of healthcare professionals. ${ }^{40}{ }^{41}$ The MoMiFer-app was established by experienced members of the Leuven Mindfulness Centre at KU Leuven and the psychiatrist and mindfulness teacher Maex. ${ }^{37}$ The exercises are spread over six consecutive modules. Each module consists of a short educational video clip (talking head) explaining the content and two audio files (between three and $45 \mathrm{~min}$ ) to guide experiential mindfulness meditation exercises. Participants can follow the different modules based on their own time schedule. The goal of the mindfulness exercises is to increase awareness of one's present-moment experience with an accepting, open, and non-judgemental attitude. Additionally, answers to frequently asked questions (FAQs) on mindfulness and fertility from patients are included in the app.

\section{Sample size}

Sample size is based on the experience sampling method (ESM) applying the 30/30 rule, used to determine sample size in multilevel modelling, which recommends sampling 30 participants with 30 observations per group. ${ }^{42}$ This sample size is known to achieve a sufficient statistical power to detect a moderate-to-large effect size for a single fixed effect. ${ }^{434}$ The effect is the change in symptoms of emotional distress as measured by ESM. The same approach was used in previous related research. ${ }^{45}$

\section{Outcomes, data collection and management}

Data will be collected using self-report questionnaires and ESM. ${ }^{46}$ We further evaluate the use of the app for the experimental group through app tracking. Table 1 represents an overview of outcomes, methods and timing of assessments.

Primary outcomes are symptoms of emotional distress and fertility-related quality of life (FertiQoL). Symptoms

Table 1 Outcomes, method(s) of assessment and timing of assessment

\begin{tabular}{|c|c|c|c|c|}
\hline \multirow{2}{*}{$\begin{array}{l}\text { Outcomes } \\
\text { Primary outcome measures }\end{array}$} & \multirow[t]{2}{*}{ Method(s) of assessment } & \multicolumn{3}{|c|}{ Timing of assessment } \\
\hline & & Baseline & 1.5 months & 3 months \\
\hline Fertility-related quality of life & Self-report Q & $x$ & $x$ & $X$ \\
\hline \multicolumn{5}{|l|}{ Secondary outcome measures } \\
\hline Repetitive negative thoughts & Self-report Q & $x$ & $x$ & $x$ \\
\hline Self-compassion & Self-report Q & $x$ & $x$ & $x$ \\
\hline User-rated quality of the app & Self-report Q & & & $\mathrm{X}$ \\
\hline Use of the app & App tracking & & $x$ & $X$ \\
\hline
\end{tabular}

ESM, experience sampling method; $Q$, questionnaire. 
of emotional distress are operationalized as symptoms of depression, anxiety and stress measured with the Depression, Anxiety and Stress Scale (DASS-21), ${ }^{47}$ and ESM. ${ }^{46}$ The DASS-21 contains a depression, anxiety and stress subscale. The overall score lies between 0 and 126 . Higher scores suggest more symptoms of emotional distress.

ESM items question symptoms of depression, anxiety, and stress with a sliding bar ranging from 0 to 100 resulting in a total score of symptoms of emotional distress. Lower scores reflect less symptoms of emotional distress. ESM is a validated, structured diary technique to assess participants in the context of their daily living environment. It is a momentary assessment method providing repeated, in-the-moment micromeasurements of core psychological and behavioural variables in a prospective and ecologically valid manner. ${ }^{46}$ The ESM questions will be administered via the app. In each of the three assessment phases, participants' smartphones, via the app, will beep 10 times/day for four consecutive days according to a semistratified interval scheme (waking hours will be divided into 10 equal intervals and in each interval one beep will be randomly programmed). At each beep, participants will be asked to indicate their current experience of emotions (eg, sad mood), RNT and mindfulness skills.

FertiQoL is surveyed with the FertiQoL questionnaire. ${ }^{48} 49$ The Core FertiQoL total score is calculated across the emotional, relational, mind-body and social subdomains and lies between 0 and 100. Higher scores indicate better FertiQoL.

Secondary outcomes are mindfulness skills, RNT, selfcompassion, user-rated quality of the app and use of the app. Mindfulness skills are measured with ESM and the Comprehensive Inventory of Mindfulness ExperiencesShort Form (CHIME-SF). ${ }^{50}$ ESM measurement encompasses questions on state mindfulness using a sliding bar. Total score varies between 0 and 100, with higher scores indicating better mindfulness skills. CHIME-SF questions aspects of mindfulness in daily life. The subscales are inner awareness, outer awareness, acting with awareness, acceptance, decentring/nonreactivity, openness, relativity and insight. The total score lies between 24 and 144, with higher scores representing better mindfulness skills. RNT is assessed with the Perseverative Thinking Questionnaire (PTQ).$^{5152}$ The PTQ covers questions on RNT containing the following subscales: Core features of RNT (repetitiveness, intrusiveness and difficulties to disengage), perceived unproductiveness of RNT, and RNT capturing mental capacity subscales. Total score ranges from 0 to 60 , with higher scores indicating more RNT. The Self-Compassion Scale-Short Form ${ }^{53-55}$ measures self-compassion through six subscales: self-kindness, common-humanity, mindfulness, selfjudgement, isolation and over-identification. Total score ranges from 12 to 60 with higher scores indicating more self-compassion.

All prior mentioned assessments will be gathered for both the experimental and the control group at baseline, and 1.5 months and 3 months after randomisation (table 1).

For the experimental group, the user-rated quality of the app will be obtained at the end of the study using the short version of the subjective quality subscale of the Mobile App Rating Scale. ${ }^{56}$ It offers questions on recommendation and scoring of the app. Total score lies between 0 and 8 , with higher scores meaning higher quality. Use of the stand-alone mobile mindfulness app will be monitored through app tracking. We will track how often participants in the experimental group open the application, which exercises they perform, and whether or not they finish the exercises.

Sociodemographic characteristics, fertility-related information (ie, fertility treatment, infertility diagnosis, causes of infertility, complementary therapy and time spent trying to get pregnant), and prior experience with mindfulness are reported by the participants during baseline measurements.

Questionnaire data will be collected using Qualtrics and ESM in the MoMiFer-app. ${ }^{46}$ Storage and analysis will be done by the study investigator in SPSS according to Good Clinical Practice (GCP). Deviation of maximally 1 week before and after the planned time point is allowed. The data from the mobile mindfulness application can be retrieved from the secured website of MoMiFer of which only the research team and the participants have access.

\section{Participant timeline}

Figure 1 provides an overview of the study procedure. The informed consent is sent to potential participants through electronic mail. Consenting people with infertility are invited for study intake through an online video call. During this standard intake additional questions regarding the study are answered and the assessment methods are explained. Once consent is given, participants complete the baseline measurements (T0). These consist of web-based questionnaires. Only after this baseline assessment, participants will be randomised to prevent bias. Next, the MoMiFer-app is installed, providing the complete stand-alone mobile mindfulness application (experimental group) or the version with solely information regarding the study and the ESM (wait-list control group). Participants receive an overview of individualised assessment moments, and reminders via the app and electronic mail to promote participant retention and complete follow-up measurements. Subsequently, participants will be asked to fill out ESM on their smartphone via the app for four consecutive days (T0). During these 4 days, participants' smartphones will beep 10 times a day to remind them (through beeps and pop-up messages) to answer a set of short questions about their mood and thoughts, which takes about $90 \mathrm{~s}$. Follow-up measurements, including ESM and similar questionnaires to the baseline assessment, take place at 1.5 (T1) and 3 months (T2) after randomisation. At T2 (ie, the end of the study) the experimental group will be asked to fill out a questionnaire about the user-rated quality of the app. 


\section{Data analysis}

Intention-to-treat analysis was performed in the statistical software program SPSS Version 27.0. Descriptive statistics on baseline characteristics will be presented for the two arms. We will use hierarchical linear modelling to examine differential trajectories. ${ }^{57}$ Estimator for missing data is full information maximum likelihood. To test the experimental effect, we will use a multilevel model with two levels: time points (Level 1) will be nested within persons (level 2). In this model, (1) the dummy-coded assessment time (as a level-1 variable), (2) the treatment condition (as a level-2 variable) and (3) their cross-level interactions are included in predicting the outcome. We will also use Cohen's d statistic to calculate within- and between-group effect sizes. A $p<0.05$ will be used to determine statistical significance for the experimental group. The attrition rate regarding enrolment and drop-out will be reported and compared between the two arms.

\section{Harms}

Expectation concerning harms for participants is minimal. No exclusion criteria are applied in the area of people at high risk (for example mental illness). ${ }^{32}$ Each participant signs an informed consent which includes a list of (mental) healthcare organisations. This information is furthermore provided at each of the three assessments. All solicited and spontaneously reported adverse events and other unintended effects of the MoMiFer study will be collected, assessed, reported and managed according to GCP guidelines.

\section{Patient and public involvement}

A human-centred design was applied for creating the MoMiFer-app by involving both patients and healthcare professionals in the development process. ${ }^{34}$ Moreover, an advisory committee was inherently part of the project, consisting of representatives of the Belgian non-profit patient organisation 'De Verdwaalde Ooievaar' and of the 'Belgian Society for Reproductive Medicine'. In addition, participants are recruited through two non-profit patient organisations: 'De Verdwaalde Ooievaar' and 'Kinderwens'. If the study indicates the MoMiFer-app adds value to reducing symptoms of emotional distress and improving FertiQoL in people experiencing infertility, the app might become available to both organisations afterwards.

\section{ETHICS AND DISSIMINATION}

This study was approved by the Ethics Committee Research UZ/KU Leuven (Belgium) (S62323). Any subsequent protocol amendments will be submitted to the appropriate Ethics Committees and national Regulatory Authorities for approval. Participants IDs are used to guarantee confidentiality of participant's data (ie, coding of dataset). The document linking the IDs to the identifiable information is stored separately. Access to coded data is only allowed for the MoMiFer research team. The investigators will disseminate results from this research through presentations at public lectures, scientific institutions and meetings, and/or publications in scientific journals. International Committee of Medical Journal Editors recommendations will be followed regarding authorship to publications. We do not intend to use professional writers.

\section{DISCUSSION}

With this exploratory RCT, we examine the impact of a stand-alone mobile mindfulness app on symptoms of emotional distress and FertiQoL of people experiencing infertility, including women, men and couples. The MoMiFer-app provides several mindfulness exercises varying in length accompanied with talking heads, and information regarding mindfulness and infertility. The app was developed at the KU Leuven following an evidence-based approach, based on qualitative data from patients with infertility and an expert panel opinion of healthcare professionals. ${ }^{41}$ An important strength of this exploratory study is the combination of self-report questionnaires with in the moment measurements through $\mathrm{ESM}^{46}$ within a repeated measures design. Second, given the smartphone-delivered, stand-alone character of the app, the MoMiFer-app can reach a broad audience in an accessible and cost-efficient way. Thereby, offering a first introduction to app-delivered mindfulness exercises. Moreover this research will focus on women, men, and couples experiencing infertility, as the majority of studies have been focusing exclusively on women with infertility. ${ }^{25} 58$ Finally, objectively evaluating the use of the app through app tracking and querying the userrated quality of the app will give insight in how people use and experience the stand-alone mobile mindfulness app. In this study, participants are self-decisive to practice mindfulness through the app to evaluate the use of the app. However, research points out that regular practice is recommended to enhance mindfulness skills and the associated outcomes. ${ }^{17293259}$ Another limitation related to the study design, is that this is an open-label trial where solely the statistician is masked. Furthermore, infertility treatment and concomitant care (eg, acupuncture, fertility coach) are allowed during the trial which may bias the outcome measures. This is taken into account in analysis as both topics are questioned during baseline measurements and at the end of the study. Future more in-depth research could integrate a third active control group as a wait-list control condition may inflate estimates of the experimental effect. ${ }^{60}$ If the MoMiFer-app would prove to be effective, it could be integrated in standard care, offering an easily accessible, low-intensive and a more cost-efficient strategy for people experiencing infertility to improve their mental health.

\section{Author affiliations}

${ }^{1}$ Department of Chronic Diseases and Metabolism, KU Leuven, Leuven, Belgium

${ }^{2}$ Leuven Mindfulness Centre, KU Leuven, Leuven, Belgium 
${ }^{3}$ Faculty of Psychology and Educational Sciences, KU Leuven, Leuven, Belgium ${ }^{4}$ Leuven University Fertility Centre, Katholieke Universiteit Leuven UZ Leuven, Leuven, Belgium

${ }^{5}$ Department of Development and Regeneration, KU Leuven, Leuven, Belgium ${ }^{6}$ Department of Endocrinology, Clinical Nutrition, Katholieke Universiteit Leuven UZ Leuven, Leuven, Belgium

\section{Twitter Christophe Matthys @NutritionObesi1}

Acknowledgements We acknowledge Steve De Backer for the technical development of the MoMiFer-app; Edel Maex, for codeveloping the stand-alone mobile mindfulness app; VZW Kinderwens, VZW De Verdwaalde Ooievaar, Praxis $P$ and multiple fertility counsellors through social media for recruitment of participants.

Contributors TB, NW, CM, ED, CS, KVdG, SLF and FR designed the trial, developed the protocol, and applied for funding. TB, NW, FR and KVdG applied for ethical approval. TB, NW and KVdG implemented the logistics of the trial. All authors read, revised and approved the final manuscript.

Funding This work was supported by KU Leuven with funding by FWO-TBM (reference: T005417N).

Competing interests KVdG and FR are founders and members of the managing committee of the Leuven Mindfulness Centre Fund. FR and KVdG receive payments for workshops and presentations related to mindfulness.

\section{Patient consent for publication Not applicable.}

Provenance and peer review Not commissioned; externally peer reviewed.

Supplemental material This content has been supplied by the author(s). It has not been vetted by BMJ Publishing Group Limited (BMJ) and may not have been peer-reviewed. Any opinions or recommendations discussed are solely those of the author(s) and are not endorsed by BMJ. BMJ disclaims all liability and responsibility arising from any reliance placed on the content. Where the content includes any translated material, BMJ does not warrant the accuracy and reliability of the translations (including but not limited to local regulations, clinical guidelines, terminology, drug names and drug dosages), and is not responsible for any error and/or omissions arising from translation and adaptation or otherwise.

Open access This is an open access article distributed in accordance with the Creative Commons Attribution Non Commercial (CC BY-NC 4.0) license, which permits others to distribute, remix, adapt, build upon this work non-commercially, and license their derivative works on different terms, provided the original work is properly cited, appropriate credit is given, any changes made indicated, and the use is non-commercial. See: http://creativecommons.org/licenses/by-nc/4.0/.

\section{ORCID iDs}

Tessy Boedt http://orcid.org/0000-0001-5417-4258

Eline Dancet http://orcid.org/0000-0003-0356-930X

Christophe Matthys http://orcid.org/0000-0003-1770-6862

\section{REFERENCES}

1 Zegers-Hochschild F, Adamson GD, Dyer S, et al. The International glossary on infertility and fertility care, 2017. Hum Reprod 2017;32:1786-801.

2 Mascarenhas MN, Flaxman SR, Boerma T, et al. National, regional, and global trends in infertility prevalence since 1990: a systematic analysis of 277 health surveys. PLoS Med 2012;9:e1001356.

3 de Geyter C, Calhaz-Jorge C, Kupka MS. Art in Europe, 2014: results generated from European registries by ESHRE. Human Reproduction 2018;33:1586-601.

4 Sullivan EA, Zegers-Hochschild F, Mansour R, et al. International Committee for monitoring assisted reproductive technologies (ICMART) world report: assisted reproductive technology 2004. Hum Reprod 2013;28:1375-90.

5 Luk BH-K, Loke AY. The impact of infertility on the psychological well-being, marital relationships, sexual relationships, and quality of life of couples: a systematic review. J Sex Marital Ther 2015;41:610-25.

6 Verhaak CM, Smeenk JMJ, van Minnen A, et al. A longitudinal, prospective study on emotional adjustment before, during and after consecutive fertility treatment cycles. Hum Reprod 2005;20:2253-60.

7 Verhaak CM, Smeenk JMJ, Evers AWM, et al. Women's emotional adjustment to IVF: a systematic review of 25 years of research. Hum Reprod Update 2007;13:27-36.
8 Huppelschoten AG, van Dongen AJCM, Verhaak CM, et al. Differences in quality of life and emotional status between infertile women and their partners. Hum Reprod 2013;28:2168-76.

9 Milazzo A, Mnatzaganian G, Elshaug AG, et al. Depression and anxiety outcomes associated with failed assisted reproductive technologies: a systematic review and meta-analysis. PLoS One 2016;11:1-19.

10 Agostini F, Monti F, Andrei F, et al. Assisted reproductive technology treatments and quality of life: a longitudinal study among subfertile women and men. J Assist Reprod Genet 2017;34:1307-15.

11 Chachamovich JR, Chachamovich E, Ezer H, et al. Investigating quality of life and health-related quality of life in infertility: a systematic review. J Psychosom Obstet Gynaecol 2010;31:101-10.

12 Domar AD, Rooney K, Hacker MR, et al. Burden of care is the primary reason why insured women terminate in vitro fertilization treatment. Fertil Steril 2018;109:1121-6.

13 Gameiro S, Boivin J, Peronace L, et al. Why do patients discontinue fertility treatment? A systematic review of reasons and predictors of discontinuation in fertility treatment. Hum Reprod Update 2012;18:652-69.

14 Kabat-Zinn J. Gezond leven Met mindfulness: handboek meditatief ontspannen. Altamira, 2014.

15 Khoury B, Lecomte T, Fortin G, et al. Mindfulness-based therapy: a comprehensive meta-analysis. Clin Psychol Rev 2013;33:763-71.

16 Spijkerman MPJ, Pots WTM, Bohlmeijer ET. Effectiveness of online mindfulness-based interventions in improving mental health: a review and meta-analysis of randomised controlled trials. Clin Psychol Rev 2016:45:102-14.

17 Carmody J, Baer RA. Relationships between mindfulness practice and levels of mindfulness, medical and psychological symptoms and well-being in a mindfulness-based stress reduction program. J Behav Med 2008;31:23-33.

18 van der Gucht K. Application to diverse populations and working mechanisms, 2017: 1-10.

19 Hosseini MS, Mousavi P, Hekmat K, et al. Effects of a short-term mindfulness-based stress reduction program on the quality of life of women with infertility: a randomized controlled clinical trial. Complement Ther Med 2020;50:1-22.

20 Nery SF, Paiva SPC, Vieira Érica L, et al. Mindfulness-based program for stress reduction in infertile women: randomized controlled trial. Stress Health 2019:35:49-58.

21 Li J, Long L, Liu Y, et al. Effects of a mindfulness-based intervention on fertility quality of life and pregnancy rates among women subjected to first in vitro fertilization treatment. Behav Res Ther 2016;77:96-104.

22 Gaitzsch H, Benard J, Hugon-Rodin J. The effect of mind-body interventions on psychological and pregnancy outcomes in infertile women: a systematic review. Archives of Women's Mental Health, 2020.

23 Ying L, Wu LH, Loke AY. The effects of psychosocial interventions on the mental health, pregnancy rates, and marital function of infertile couples undergoing in vitro fertilization: a systematic review. J Assist Reprod Genet 2016;33:689-701.

24 Galhardo A, Cunha M, Pinto-Gouveia J. Mindfulness-Based program for infertility: efficacy study. Fertil Steril 2013;100:1059-67.

25 Gaitzsch H, Benard J, Hugon-Rodin J, et al. The effect of Mindbody interventions on psychological and pregnancy outcomes in infertile women: a systematic review. Arch Womens Ment Health 2020;23:479-91.

26 Galhardo A, Cunha M, Pinto-Gouveia J. A 7-year follow-up study of the Mindfulness-Based program for infertility: are there long-term effects? Clin Psychol Psychother 2019;26:409-17.

27 Bai C-F, Cui N-X, Xu X, et al. Effectiveness of two guided selfadministered interventions for psychological distress among women with infertility: a three-armed, randomized controlled trial. Hum Reprod 2019;34:1235-48.

28 Hosseini MS, Mousavi P, Hekmat K, et al. Effects of a short-term mindfulness-based stress reduction program on the quality of life of women with infertility: a randomized controlled clinical trial. Complement Ther Med 2020;50:102403.

29 Linardon J, Cuijpers P, Carlbring P, et al. The efficacy of appsupported smartphone interventions for mental health problems: a meta-analysis of randomized controlled trials. World Psychiatry 2019;18:325-36.

30 S S, Cristea IA. The efficacy of mindfulness meditation apps in enhancing users' well-being and mental health related outcomes: a meta-analysis of randomized controlled trials. J Affect Disord 2020.

31 Jayewardene WP, Lohrmann DK, Erbe RG, et al. Effects of preventive online mindfulness interventions on stress and mindfulness: a metaanalysis of randomized controlled trials. Prev Med Rep 2017;5:150-9. 
32 Linardon J. Can acceptance, mindfulness, and Self-Compassion be learned by smartphone Apps? A systematic and meta-analytic review of randomized controlled trials. Behav Ther 2020;51:646-58

33 Van Dongen AJCM, Kremer JAM, Van Sluisveld N, et al. Feasibility of screening patients for emotional risk factors before in vitro fertilization in daily clinical practice: a process evaluation. Hum Reprod 2012;27:3493-501.

34 Boedt T, Dancet E, Lie Fong S. Systematic development of a mobile preconception lifestyle programme for couples undergoing in vitro fertilisation: the PreLiFe-programme. BMJ Open.

35 Chan A-W, Tetzlaff J, Altman D. Research and Reporting Methods Annals of Internal Medicine SPIRIT 2013 Statement : Defining Standard Protocol Items for Clinical Trials. Ann Intern Med 2013;158:200-7.

36 Hallingberg B, Turley R, Segrott J, et al. Exploratory studies to decide whether and how to proceed with full-scale evaluations of public health interventions: a systematic review of guidance. Pilot Feasibility Stud 2018;4:1-12.

37 Maex E. Leven in de maalstroom. Lannoo, 2006.

38 Crane RS, Brewer J, Feldman C, et al. What defines mindfulnessbased programs? the warp and the weft. Psychol Med 2017;47:990-9.

39 Boedt T, Dancet E, Lie Fong S. Effectiveness of a mobile preconception lifestyle programme in couples undergoing in vitro fertilisation (IVF): the protocol for the PreLiFe randomised controlled trial (PreLiFe-RCT). BMJ Open 2019;9:1-8.

40 Craig P, Dieppe P, Macintyre S, et al. Developing and evaluating complex interventions: the new medical Research Council guidance. BMJ 2008;337:979-83.

41 Medical Research Council. A framework for development and RCTs for complex interventions, 2000: 1-19. http://www.mrc.ac.uk/Utilities/ Documentrecord/index.htm?d=MRC003372

42 Hox JJ, Moerbeek M, van de Schoot R. Multilevel analysis: techniques and applications. Third ed. Routledge, 2017.

43 Scherbaum CA, Ferreter JM. Estimating statistical power and required sample sizes for organizational research using multilevel modeling. Organ Res Methods 2009;12:347-67.

44 Mathieu JE, Aguinis H, Culpepper SA, et al. Understanding and estimating the power to detect cross-level interaction effects in multilevel modeling. J Appl Psychol 2012;97:951-66.

45 Van der Gucht K, Dejonckheere E, Erbas Y, et al. An experience sampling study examining the potential impact of a mindfulnessbased intervention on emotion differentiation. Emotion 2019;19:123-31.
46 Csikszentmihalyi M. Flow and the foundations of positive psychology, 2014.

47 de Beurs E, van Dyck R, Lange A. De DASS: Een vragenlijst voor Het meten van depressie, angst en stress. Gedragstherapie 2001;34:35-53

48 Aarts JWM, van Empel IWH, Boivin J, et al. Relationship between quality of life and distress in infertility: a validation study of the Dutch FertiQoL. Hum Reprod 2011;26:1112-8.

49 Boivin J, Takefman J, Braverman A. The fertility quality of life (FertiQoL) tool: development and general psychometric properties. Hum Reprod 2011;26:2084-91.

50 Cladder-Micus MB, Verweij $\mathrm{H}$, van Ravesteijn $\mathrm{H}$, et al. Validation of the Dutch comprehensive inventory of mindfulness experiences (CHIME) and development of a short form (CHIME-SF). Mindfulness 2019;10:1893-904

51 Ehring T, Zetsche U, Weidacker K, et al. The perseverative thinking questionnaire (PTQ): validation of a content-independent measure of repetitive negative thinking. J Behav Ther Exp Psychiatry 2011;42:225-32.

52 Ehring T, Raes F, Weidacker K, et al. Validation of the Dutch version of the perseverative thinking questionnaire (PTQ-NL). Eur J Psychol Assess 2012;28:102-8.

53 Raes F, Pommier E, Neff KD, et al. Construction and factorial validation of a short form of the Self-Compassion scale. Clin Psychol Psychother 2011;18:250-5.

54 Neff KD. The Self-Compassion scale is a valid and theoretically coherent measure of Self-Compassion. Mindfulness 2016;7:264-74.

55 Raes F, Pommier E, Neff KD, et al. Construction and factorial validation of a short form of the Self-Compassion scale. Clin Psychol Psychother 2011;18:250-5.

56 Stoyanov SR, Hides L, Kavanagh DJ, et al. Mobile APP rating scale: a new tool for assessing the quality of health mobile apps. JMIR Mhealth Uhealth 2015;3:e27.

57 Raudenbush SW, Bryk AS. Hierarchical linear models: applications and data analysis methods. Second ed. SAGE Publications, 2001.

58 Ying LY, Wu LH, Loke AY. Gender differences in experiences with and adjustments to infertility: a literature review. Int J Nurs Stud 2015;52:1640-52.

59 Krusche A, Cyhlarova E, Williams JMG. Mindfulness online: an evaluation of the feasibility of a web-based mindfulness course for stress, anxiety and depression. BMJ Open 2013;3:1-10.

60 Cunningham JA, Kypri K, McCambridge J. Exploratory randomized controlled trial evaluating the impact of a waiting list control design. BMC Med Res Methodol 2013;13:1-7. 